\title{
Clinical profile and outcome of patients with placenta previa: a study at a tertiary care referral institute in Northern India
}

\author{
Ekta Jaiswal ${ }^{1 *}$, Neelam Aggarwal ${ }^{2}$, Vanita Suri $^{2}$, Rashmi Bagga ${ }^{2}$, \\ Jasvinder Kalra ${ }^{2}$, Pradeep Gupta ${ }^{3}$
}

\begin{abstract}
${ }^{1}$ Department of Obstetrics and Gynaecology, Patan Academy of Health Sciences, Kathmandu, Nepal
${ }^{2}$ Department of Obstetrics and Gynaecology, PGIMER, Chandigarh, India

${ }^{3}$ Department of Paediatrics, Siddhi Memorial Hospital, Bhaktapur, Nepal
\end{abstract}

Received: 25 April 2018

Accepted: 25 May 2018

\section{*Correspondence:}

Dr. Ekta Jaiswal,

E-mail: ektaaa12@gmail.com

Copyright: (C) the author(s), publisher and licensee Medip Academy. This is an open-access article distributed under the terms of the Creative Commons Attribution Non-Commercial License, which permits unrestricted non-commercial use, distribution, and reproduction in any medium, provided the original work is properly cited.

\begin{abstract}
Background: The aim of this study was to determine clinical profile, evaluate our antenatal and intraoperative management and see the maternal and perinatal outcome in patients with placenta previa.

Methods: A prospective study was carried out in 130 women with placenta previa in the Department of Gynecology, PGIMER, Chandigarh, India between Jan 2015-April 2016. The profile of these patients was recorded in a predesigned proforma and maternal and perinatal outcome analyzed in detail.

Results: One third (46/130) of the patients with placenta previa had a history of previous caesarian section, $27 \%$ had previous uterine curettage and $82 \%$ were multiparous. $18 \%$ were asymptomatic placenta previa whereas $82 \%$ had one or more bleeding episodes. Expectant management was given to $67 \%$ patients after first bleeding episode. Majority (92/130) of patients required emergency cesarean section. Due to invasive placentation, 25 patients required cesarean hysterectomy. Ninety percent patients required delivery at $\leq 37$ weeks and neonatal outcome improved with increasing gestation as expected.

Conclusions: Reduction in cesarean rate is the major key factor for decreasing the incidence of placenta previa as, as well as placenta accreta and other associated complications as there were no patients diagnosed to have placenta accreta when placenta previa was present without any previous cesarean scar. In cases of invasive placenta, performing a classical CS, not trying to remove the placenta and proceeding directly to hysterectomy resulted in reduced blood loss. Neonatal outcome as well as maternal outcome is best when cesarean is done between 36-37 weeks.
\end{abstract}

Keywords: Adherent placenta, Cesarean section, Hysterectomy, Placenta previa

\section{INTRODUCTION}

Placenta previa is a condition in which placenta is implanted in the lower segment of the uterus. It may completely or partially obstruct the internal os of the cervix. Placenta previa occurs in approximately 5 in
1,000 pregnancies. ${ }^{1}$ It is a major cause of third-trimester hemorrhage.

The etiology of placenta previa is speculative but there are several risk factors associated with this condition. These include advanced maternal age, multiparity, closely spaced pregnancies, previous cesarean deliveries 
or uterine surgeries, maternal smoking, use of artificial reproductive technologies, recurrent spontaneous or elective abortions and history of curettage, a male fetus, etc. Women with placenta previa are likely to have more complications and adverse perinatal outcomes than those with normally implanted placenta. Adverse maternal and neonatal outcomes like antenatal and intra-partum hemorrhage, need of blood transfusion and emergency hysterectomy, intra-uterine growth restriction and preterm birth are usually seen with complete placenta previa.

According to RCOG guidelines, the timing of delivery should be based on clinical scenario supplemented by sonographic information. In asymptomatic women, elective delivery by cesarean section is not recommended before 38 weeks of gestation for placenta previa, or before 36-37 weeks of gestation for suspected placenta accreta. $^{2}$

Since there are no clear-cut guidelines on timing of cesarean in cases of placenta previa especially in asymptomatic women as well as stable women who had bled earlier and, in such patients, waiting for one extra week would help in improved perinatal outcome, authors planned this prospective study to know the clinical profile and outcome of pregnancies complicated by placenta previa in present set up.

\section{METHODS}

This prospective study was conducted in the Department of Obstetrics and Gynecology, PGIMER, Chandigarh from Jan 2015 to April 2016. This study was approved by the Institutional Ethics Committee of Post-graduate Institute of Medical Education and Research (PGIMER), Chandigarh. A total of 130 patients diagnosed with placenta previa on ultrasound were included in the study, since the time of diagnosis. The data was collected after explaining the study protocol and informed written consent. As the study did not involve any extra intervention, none of the women denied. Cases were managed according to institute protocol. All the collected data was transferred to predesigned proforma and data was analyzed using SPSS (version 12) and Excel 2010 software.

\section{RESULTS}

\section{Demographics}

The mean age of patients presenting with placenta previa was $28.5 \pm 4.6$ years with minimum age of presentation being 19 years and maximum being 45 yrs. $18 \%$ were primigravida. Five among 130 patients had recurrent placenta previa among which $2 / 5$ had adherent placenta. One-third patient had previous CS. History of previous abortion was present in $42(32 \%)$ patients among which $35 / 42$ patients had previous history of dilatation and curettage done. Posterior placentation was more common than anterior placentation (Table 1).

Table 1: Demographic details of study patients.

\begin{tabular}{|ll|}
\hline Parameter & Value \\
\hline Age $($ Mean \pm SD) years & $28.5 \pm 4.6$ \\
\hline Gravidity (Mean \pm SD) & $2.62 \pm 1.2$ \\
\hline Parity (Mean \pm SD) & $1 \pm 1$ \\
\hline H/O previous surgical abortion & $35(27 \%)$ \\
\hline Recurrent placenta previa & $5(3.8 \%)$ \\
\hline H/O cesarean section & $46(35.4 \%)$ \\
\hline Previous 1 CS & $30(23.1 \%)$ \\
\hline Previous 2 CS & $13(10 \%)$ \\
\hline Previous 3 CS & $3(2.3 \%)$ \\
\hline Anterior placenta & $62(47.7 \%)$ \\
\hline Posterior placenta & $68(52.3 \%)$ \\
\hline
\end{tabular}

\section{Antenatal USG assessment}

Most of the placenta previas were diagnosed by trans abdominal scan however 51 underwent additional TVS to confirm the abnormal placental site in the third trimester. Among the 51 patients of placenta previa who underwent TVS, 21 patients had posterior placenta. Among the enrolled patients ,19/130 were found to have adherent placenta in either USG or MRI. Only a small number (7/130) of patients had undergone magnetic resonance imaging.

\section{Hospital admission}

The mean period of gestation for hospital admission in patients with placenta previa was $33 \mathrm{wks} \pm 3$ weeks while in asymptomatic placenta previa it was 35.25 \pm 1.82 weeks. Most common reason for hospital admission was bleeding per vaginum $(70.8 \%)$; pre-labor rupture of membranes in $2.3 \%$ and logistics (distance from hospital or lack of support from home) in $16.9 \%$. Ten percent were admitted due to other causes like abruption, eclampsia, pain abdomen etc. Majority (106/130) of the patients had one or more per vaginal bleeding episodes after 20 weeks period of gestation. The mean period of gestation of first bleeding episode was $24 \pm 7$ weeks. The average number of bleeding episodes among the patients who had bleeding was $2 \pm 2$ episodes.

Out of 24 patients with asymptomatic placenta previa, 5 had adherent placenta (accreta). Among 106 patients, during the first bleeding episode, $58 \%$ received two doses of steroids (Betamethasone / Dexamethasone) whereas $22 \%$ received progesterone (intramuscular). Majority $(87 / 106)$ of patients received expectant management after the first bleeding episode while the rest 19 patients required emergency termination of pregnancy due to severe bleeding. Those who received expectant management gained a mean duration of 37 days \pm 43 days. Among those who received expectant management, 73 required emergency $\mathrm{CS}$ while 14 had elective CS done. 


\section{Intraoperative details}

There was equal number of patients receiving spinal anesthesia $(n=65)$ and general anesthesia $(n=65)$ during the cesarean section. Lower segment cesarean section was performed in $82 \%$ of the patients where as $18 \%$ patients had classical cesarean section done. Two patients had hysterotomy done. Around $90 \%$ patients had preterm delivery. A total of $41.5 \%$ patients were needed to be delivered in early preterm period i.e. before 34 weeks gestation; $50 \%$ between 34 and 37 weeks and only $8.5 \%$ had term cesarean section i.e. $\geq 37$ weeks gestation. The mean period of gestation at delivery was $35 \pm 3$ weeks; minimum being 20 weeks which was a hysterotomy resulting in a 400 grams abortus and maximum being 41 weeks which was a referred case of placenta previa to present institute at $40+6$ weeks.

\section{Intraoperative complications and hemostasis}

Various intraoperative complications like postpartum hemorrhage and need of blood transfusion, bladder injury, need of ICU admission was analyzed separately. In two patients, placenta could not be removed completely so it was left to involute. The mean amount of blood loss during CS was $886 \pm 877 \mathrm{ml}$; minimum being $200 \mathrm{ml}$ and maximum being $5500 \mathrm{ml}$.The average number of units of blood transfusion was $1.3 \pm 2.4$ units with the maximum number of blood units transfused being 18 units. There was more blood loss during the cesarean section of patients with adherent placenta around 2litres compared to patients with non-adherent placenta around $650 \mathrm{ml}$. Bladder injury (serosal) occurred in 5 patients. One patient had mortality after 2 days of ICU stay. She was G2P1001 with k/c/o placenta accreta. She had blood loss of $5500 \mathrm{ml}$ and had received 18 units of blood transfusion. Eleven patients were near miss as they received 5 or more units of blood transfusion and 8/11 patients also needed intubation and ventilation not related to anesthesia.

During cesarean section no additional steps were required for achieving hemostasis other than the routine steps followed during CS in 54 patients whereas 76/130 required additional methods to achieve hemostasis (Table 2). Unilateral ligation of uterine artery was done in 18 patients whereas 2 patients required internal iliac artery ligation to control bleeding. Bakri balloon was placed in 6 patients whereas 25 patients required hysterectomy: 14 for accreta, 8 for percreta and 3 for PPH.

The incidence of adherent placenta increased with the number of previous CS: $36.7 \%, 61.5 \%$ and $100 \%$ after 1 , 2 and 3 previous CS respectively. Among 22 adherent placentas, 19 were antenatally diagnosed by various imaging modalities. In those $17 / 19$ patients, classical CS was performed without attempting to remove the placenta followed by hysterectomy which resulted in reduced blood loss $(1900 \pm 1057.7 \mathrm{ml})$ compared to doing LSCS, trying to remove the placenta and then proceeding to hysterectomy in the remaining 2 patients $(2500 \pm 707.1 \mathrm{ml})$.

Table 2: Intraoperative complications and methods of hemostasis.

\begin{tabular}{|l|l|}
\hline Details & $(\mathrm{n}=130)(\%)$ \\
\hline PPH & $32(24.6 \%)$ \\
\hline Invasive placentation & $22(16.9 \%)$ \\
\hline Incomplete removal of placenta & $2(1.5 \%)$ \\
\hline Need of blood transfusion & $61(46.9 \%)$ \\
\hline Bladder injury & $5(3.84 \%)$ \\
\hline ICU admission & $10(7.7 \%)$ \\
\hline Near miss & $11(8.5 \%)$ \\
\hline Mortality & $1(0.8 \%)$ \\
\hline No additional step & $54(41.5 \%)$ \\
\hline Placental bed sutures & $51(39.2 \%)$ \\
\hline Ligation of uterine artery & $18(13.8 \%)$ \\
\hline Ligation of internal iliac artery & $2(1.5 \%)$ \\
\hline Placement of Bakri balloon & $6(4.6 \%)$ \\
\hline Hysterectomy & $25(19.3 \%)$ \\
\hline
\end{tabular}

\section{Neonatal outcome}

Out of total 130 babies born, 127 were live born while 3 babies were still born at 20, 28 and 34 weeks respectively (Table 3). The mean birth weight of babies born was $2.18 \pm 0.63 \mathrm{~kg}$. Minimum birth weight was 400 grams whereas maximum was $3.4 \mathrm{~kg}$. Among them ,14 babies had APGAR $<7$ at 5 minutes. $17.7 \%$ required admission in NICU due to premature delivery. Out of 130 babies born 3 had congenital malformation. One of them had hypospadias, other had meningocele and last one had short femur.

Table 3: Neonatal outcome of study population.

\begin{tabular}{|c|c|c|c|}
\hline Parameters & & $\begin{array}{l}\text { Number } \\
(n-130)\end{array}$ & Percentage \\
\hline \multirow{2}{*}{ LB/SB } & Live birth & 127 & 97.7 \\
\hline & Stillbirth & 3 & 2.3 \\
\hline \multirow{3}{*}{$\begin{array}{l}\text { Maturity } \\
\text { (weeks) }\end{array}$} & $<34$ & 54 & 41.5 \\
\hline & 34- 37 & 65 & 50 \\
\hline & $>37$ & 11 & 8.5 \\
\hline \multirow{3}{*}{$\begin{array}{l}\text { Weight of } \\
\text { babies (kg) }\end{array}$} & $<1.5$ & 16 & 12.3 \\
\hline & $1.5-2.5$ & 36 & 27.7 \\
\hline & $>2.5$ & 78 & 60 \\
\hline \multirow{2}{*}{ Apgar } & $<7$ at $1 \mathrm{~min}$ & 35 & 26.9 \\
\hline & $<7$ at $5 \mathrm{~min}$ & 14 & 10.8 \\
\hline \multirow{2}{*}{$\begin{array}{l}\text { Need of } \\
\text { NICU stay }\end{array}$} & Yes & 23 & 17.7 \\
\hline & No & 107 & 82.3 \\
\hline \multirow{2}{*}{$\mathrm{CMF}$} & Yes & 3 & 2.3 \\
\hline & No & 127 & 97.7 \\
\hline
\end{tabular}

\section{DISCUSSION}

Hemorrhage in pregnancy is still one of the commonest cause of maternal death in low income countries and symptomatic placenta previa can cause excessive 
bleeding risking the life of both mother and baby. The mean age of patients presenting with placenta previa in present study was $28.5 \pm 4.6$ years which is similar to the study by O. Asicioglu et al. who found the mean age to be $29.2 \pm 5.6$ years in their study of 318 patients with placenta previa. ${ }^{3}$ It is more common with advancing age as intra-myometrial and endometrial arteries deteriorate impairing normal placental development. Most (82\%) of patients with placenta previa were multi-gravidae in present study. Frequency of placenta previa increases as the parity increases remarkably and multiparty has been linked as a well-known independent risk factor for developing placenta previa.

Authors found that $35.4 \%$ patients with placenta previa had history of previous cesarean. In the study by $\mathrm{O}$. Asicioglu et al. it was found that $45 \%$ patients with placenta previa had history of previous cesarean done while in the study by T.H. Hung et al. which was done in 457 patients with placenta previa in Asian population, $11.4 \%$ patients with placenta previa had history of previous cesarean done. ${ }^{4}$

Authors can associate this finding with defects caused by previous scarring in the endometrium by previous CS. ${ }^{5}$ The majority of the previa in present study were posterior (53\%), which was similar to the study of BC Young et al. who studied 285 cases of placenta previa and found that $69 \%$ had posterior placenta previa, as evidence also suggests persistence of low lying posterior previa due to less expansion of the posterior wall compared with the anterior uterine wall. ${ }^{6,7}$

TVS is superior to TAS in diagnosing placenta previa especially in cases of obesity, under filled bladder and posterior placenta previa, in present study only 51 patients had TVS done for diagnosis of placenta previa. According to RCOG, patients with placenta previa may be managed as an outpatient, if there has been no major bleeding; a hospital nearby and a companion at most times with the woman.

In present study, most $(84 \%)$ admissions were due to bleeding, but some were admitted because of long distance from the hospital, lack of companion at all times, malpresentation of fetus, etc. After the first bleeding episode, $81 \%$ patients received expectant management to improve the neonatal mortality and morbidity related to premature delivery. The time gained by expectant management in present study was nearly 5 weeks which is good for both the mother and the fetus. According to a study by Brenner WE et al. after conservative management, prolongation of pregnancy by an average of 4 weeks after the initial bleed is seen, although significant prolongation of pregnancy is less likely when pregnancy is closer to 36 weeks' gestation.

There were equal number of patients receiving general and spinal anesthesia though spinal anesthesia is considered to be safer as inhaled anesthetics in general anesthesia cause uterine relaxation and atonic postpartum hemorrhage may occur from the placental implantation site. Kocaoglu et al. in his study says that, in absence of abnormal invasive placentation regional anesthesia is safe. $^{8}$ The majority $(92 / 130)$ of patients with placenta previa among the enrolled patients had emergency cesarean section and only 38 had elective CS which is different from the study by O.Asicioglu et al. in which among 318 patients with placenta previa, 130 had emergency cesarean section done and 188 had elective CS done. ${ }^{3}$

In present study, elective CS included the 24 patients among 130 patients who were asymptomatic stable placenta previas. This difference might be because present institute is the only tertiary referral centers for so many hospitals in this part of our country and lack of adequate knowledge in patients about the consequences of placenta previa as many patients presented for the first time directly to labour room after severe bleeding episode.

According to a recent decision analysis, optimal maternal and neonatal outcomes for women with placenta previa would be by administering steroid at 35 weeks 5 days followed by cesarean delivery at 36 weeks without amniocentesis. ${ }^{9}$ In present study, around $90 \%$ patients had preterm delivery i.e. before 37 weeks. In a study by Rosenberg et al. who studied 771 patients with placenta previa, peripartum hysterectomy was done in $5.3 \%$ among which $3 \%$ were having adherent placenta but in present study $19.3 \%$ required peripartum hysterectomy. ${ }^{10}$ Such high rate of peripartum hysterectomy can be attributed to the higher rate of referral in present hospital being a tertiary referral center and most of the adherent placentas are being referred here.

In $86.4 \%$ (19/22) peripartum hysterectomy was done for adherent placenta while in 3 patients only it was done for PPH. Rosenberg et al. have described rate of PPH and need of blood transfusion during the CS being $1.4 \%$ and $22 \%$ respectively whereas in the present study it was $25 \%$ and $47 \%$ respectively. Such vast difference can again be attributed to the large number of referrals in present institute. In patients with massive obstetrical hemorrhage, development of a disseminated intravascular coagulation (DIC) should be considered. Clotting studies and a platelet count should be done urgently and whilst awaiting the results of the coagulation studies up to 4 units of FFP and 10 units of cryoprecipitate may be given. ${ }^{11}$

In a study by Silver et al, it was observed that in the presence of placenta previa, the risk of placenta accreta was $3 \%, 11 \%, 40 \%, 61 \%$ and $67 \%$ for the first, second, third, fourth and fifth or greater repeat cesarean deliveries, respectively. In present study incidence of adherent placenta with increasing number of previous CS was: $38 \%, 62 \%$ and $100 \%$ after 1,2 , and 3 previous CS respectively. There were no patients diagnosed to have 
adherent placenta when placenta previa was present without any previous cesarean scar. A uterine scar in the lower segment attracts lower implantation of the placenta and decidua is scanty in this lower uterine segment.

In the present study, nearly $98 \%$ babies were live born which shows the good management of patients with placenta previa at present hospital. Among the enrolled patients, $91.5 \%$ babies were delivered at gestational age $<37$ weeks. The mean period of gestation at delivery was 35 weeks \pm 3 weeks in present study and the mean birth weight of babies born was $2.18 \pm 0.63 \mathrm{~kg}$ whereas in a study by Mohamed Alkhatim Alsammani et al. ${ }^{12}$ the mean period of gestation at delivery was 37weeks \pm 1 weeks whereas the mean birth weight of babies born was $3.0 \pm 0.28 \mathrm{~kg}$.

The present institute had delivery at earlier gestation due to the large number of referrals with significant bleeding at early gestation. M Schneiderman et al. did a cohort study which compiled 3,550,842 deliveries comparing neonatal outcomes of babies born to mothers with placenta previa beyond 37 weeks gestation to those delivered to other indications it was found that placenta previa was an independent risk factor for adverse neonatal outcomes. ${ }^{13}$ When delivered at term the statement that presence of placenta previa itself increases adverse neonatal outcomes becomes justifiable. But many authors do not believe that placenta previa is an independent risk factor for impaired fetal growth so there should not be significant difference between birth weight in neonates born to mothers with placenta previa and those delivered to normally located placenta. The rate of NICU admission in babies born to patients with placenta previa in present study was $18 \%$ which was similar the rate of NICU admission in the study by Mohamed Alkhatim Alsammani et al. which was $17 \%$.

\section{CONCLUSION}

From the present study authors conclude that placenta previa poses a great threat to mother as an independent risk. A multidisciplinary team must be present at the time of delivery and these patients should be delivered in a tertiary centre. Placenta accreta risk is more with more number of previous LSCS. So, it is extremely important to have higher threshold for doing cesarean in a primigravida. Reduction in cesarean rate in primigravida is the major key factor for decreasing the incidence of placenta previa as such and placenta accreta and other associated complications as well.

\section{ACKNOWLEDGMENTS}

Authors would like to thank Dr. Neelam Aggarwal, Associate Professor, Department of Obstetrics and Gynaecology, PGIMER; Dr. Vanita Suri, Professor and Head, Department of Obstetrics and Gynaecology, PGIMER; Dr. Jasvinder Kalra, Professor, Department of Obstetrics and Gynaecology, PGIMER and Dr. Rashmi
Bagga, Professor, Department of Obstetrics and Gynaecology, PGIMER for their support.

Funding: No funding sources

Conflict of interest: None declared

Ethical approval: An independent ethics committee of PGIMER, Chandigarh

\section{REFERENCES}

1. Cresswell JA, Ronsmans C, Calvert C, Filippi V. Prevalence of placenta praevia by world region: a systematic review and meta-analysis. Trop Med Int Heal. 2013 Jun;18(6):712-24.

2. Royal College of Obstetricians and Gynaecologists (RCOG) (2011). Placenta Praevia and Placenta Praevia Accreta: Diagnosis and Management. London, UK: Royal College of Obstetricians and Gynaecologists; Green-top Guideline No. 27.

3. Asıcıoglu O, Şahbaz A, Güngördük K, Yildirim G, Asicioglu BB, Ülker V. Maternal and perinatal outcomes in women with placenta praevia and accreta in teaching hospitals in Western Turkey. J Obstet Gynaecol. 2014 Aug;34(6):462-6.

4. Hung TH, Hsieh CC, Hsu JJ, Chiu TH, Lo LM. Risk factors for placenta previa in an Asian population. Int J Gynecol Obstet. 2007 Apr 1;97(1):26-30.

5. Law LW, Chor CM, Leung TY. Use of hemostatic gel in postpartum hemorrhage due to placenta previa. Obstet Gynecol. 2010;116:528-30.

6. Young BC, Nadel A and Kaima A. Does previa location matter? Surgical morbidity associated with location of a placenta previa. J Perinatol. 2014;34:264-7.

7. Cho JY, Lee YH, Mon MH, Lee JH. Difference in migration of placenta according to the location and type of placenta praevia. J Clin Ultrasound. 2008;36:79-84.

8. Kocaoglu N, Gunusen I, Karaman S, Ergenoglu AM, Firat V. Management of Anesthesia for cesarean section in parturients with placenta praevia with/without placenta accreta. Ginekologia Polska. 2012;83:99-103.

9. Zlatnik MG, Little SE, Kohli P, Kaimal AJ, Stotland NE, Caughey AB. When should women with placenta previa be delivered? A decision analysis. The Journal of reproductive medicine. 2010;55(910):373-81.

10. Rosenberg T, Pariente G, Sergienko R, Wiznitzer A, Sheiner E. Critical analysis of risk factors and outcome of placenta previa. Arch Gynecol Obstetr. $2011 \mathrm{Jul} ; 284(1): 47-51$.

11. Royal College of Obstetricians and Gynaecologists (RCOG) (2011). Antepartum haemorrhage. London, UK: Royal College of Obstetricians and Gynaecologists; Green-top Guideline No. 63.

12. Ahmed SR, Aitallah A, Abdelghafar HM, Alsammani MA. Major placenta previa: rate, maternal and neonatal outcomes experience at a 
tertiary maternity hospital, sohag, Egypt: a prospective study. JCDR. 2015 Nov;9(11):QC17.

13. Schneiderman M, Balayla J. A comparative study of neonatal outcomes in placenta previa versus cesarean for other indication at term. The J Maternal-Fetal Neonatal Med. 2013 Jul 1;26(11):1121-7.
Cite this article as: Jaiswal E, Aggarwal N, Suri V, Bagga R, Kalra J, Gupta P. Clinical profile and outcome of patients with placenta previa: a study at a tertiary care referral institute in Northern India. Int J Reprod Contracept Obstet Gynecol 2018;7:2559-64. 